\title{
LAS ORGANIZACIONES DE LA SOCIEDAD CIVIL EN FRONTERAS DE ALTA MIGRACIÓN. ENTRE EL HUMANITARISMO Y LA DEFENSA DE DERECHOS
}

\author{
Civil society organizations in high-immigration borders. \\ Between humanitarianism and the defense of rights
}

\author{
María Isolda Perelló Carrascosa* \\ Joan Lacomba**
}

\begin{abstract}
Resumen. Este artículo tiene como objetivo analizar el papel de las Organizaciones de la Sociedad Civil (OSC) en lugares de frontera con una alta migración, como son los casos de la frontera México-Estados Unidos y Marruecos-España, dos de los corredores migratorios de mayor importancia en el mundo. En estos espacios, sometidos a una vigilancia y una militarización cada vez mayores, las OSC vienen desarrollando desde hace tiempo una labor que se enfrenta crecientemente a los condicionantes impuestos por las políticas de control migratorio y de fronteras de los Estados. El artículo se basa en los resultados de del trabajo de terreno desarrollado en la ciudad fronteriza de Tijuana (México) y en las ciudades autónomas de Ceuta y Melilla (España). Nuestras investigaciones muestran cómo los niveles de actuación de las OSC en relación con las personas migrantes en tránsito, deportadas y refugiadas se mueven entre la ayuda humanitaria y la defensa de los derechos, aunque las políticas de control de flujos limitan cada vez más la acción de las organizaciones, en especial de aquellas con posicionamientos más críticos.
\end{abstract}

Palabras clave: Organizaciones de la Sociedad Civil; fronteras; derechos; ayuda humanitaria; Estados.

Abstract. The purpose of this article is analyzing the role of Civil Society Organizations (CSO) in places of high-immigration borders, such as the Mexico-United States and the Morocco-Spain borders, two of the most important migration corridors in the world. In these places, and under an increasing surveillance and militarization, CSO have for some time been carrying out work that constantly faces constraints imposed by state

\footnotetext{
Universidad de Valencia. Valencia, España. E-mail: isoldapc@gmail.com. Orcid: 0000-00023682-0356.

** Universidad de Valencia. Valencia, España. E-mail: Joan.Lacomba@uv.es. Orcid: 0000-00021067-539X.
} 
immigration and border control policies. The article is based on the results of the field work undertaken at the border city of Tijuana (Mexico) and the Autonomous Cities of Ceuta and Melilla (Spain). Our researches show how the actions of CSO in relation to immigrants in transit, deportees and refugees oscillate between humanitarian help and the defense of rights, even when flow control policies increasingly limit the work of the organizations - especially those that have a more critical stance.

Keywords: Civil Society Organizations; borders; rights; human aid; states.

\section{Introducción}

El papel de las Organizaciones de la Sociedad Civil (OSC) y su posicionamiento respecto a los Estados se ha convertido en un tema central de discusión en el ámbito de las ciencias sociales ${ }^{1}$. Desde que Hirschman formulara en 1970 su famosa propuesta en torno a los tres posibles posicionamientos ante el poder (salida, voz y lealtad), las organizaciones sociales han sido sometidas a un intenso escrutinio. Con posterioridad, los trabajos de Salamon (1994) fueron quizás los que generaron un mayor interés por las iniciativas sociales que él agrupó bajo el paraguas del Tercer Sector, abriendo las puertas a una larga serie de estudios que han venido evaluando las relaciones entre el Estado y la sociedad civil.

Salamon, que hablaba de una "revolución asociativa global" vino a poner de relieve la efervescencia asociativa y el papel creciente del Tercer Sector frente al Estado, pero también los riesgos del protagonismo asumido por la sociedad civil y su dimensión sustitutiva del propio Estado. Él mismo señaló que en esa creciente asunción de responsabilidades por parte de las organizaciones, éstas podían acabar promoviendo bien el activismo cívico, bien el voluntarismo, la profesionalización o la mercantilización de las mismas (managerialism) (Salamon, Hems, Chinnock, 2000, p. 25).

Con la perspectiva de los años, hemos podido ver cómo -de forma genérica- las organizaciones sociales no han sustituido a los Estados, pero sí se han producido transformaciones en el seno de éstas y se han establecido nuevas formas de relación entre ellas y los poderes públicos. Ya en 1994, Minkoff concluyó que se había producido una transición, en las organizaciones sociales de los Estados Unidos, desde la provisión de servicios a la defensa de derechos; y, más tarde, en 2002, él mismo señaló que en ese proceso de transición también se produjeron formas de hibridación en organizaciones que habrían asumido ambos tipos de funciones: la prestación de servicios y la defensa de derechos. De este modo, como se habría podido apreciar

1 La terminología empleada para referirse a las entidades sociales sin ánimo de lucro es muy variada, y se ha hablado de asociaciones, Organizaciones No Gubernamentales o del Tercer Sector. Aquí optamos por el término Organizaciones de la Sociedad Civil. 
de modo más reciente, las nuevas formas de hibridación, que combinan la provisión con la acción política, habrían podido dotar de un mayor éxito y continuidad en el tiempo a las organizaciones, permitiéndoles un mayor anclaje en las comunidades, pero con el contrapeso de tensiones importantes para mantener ambas funciones, marcadas por intereses divergentes (McMullin, Skelcher, 2018).

Si hablamos de las organizaciones sociales que desarrollan su acción en el campo de las migraciones -calificadas habitualmente como organizaciones pro-migrantes-, también podríamos hacer referencia a la incidencia de procesos similares a los señalados por los anteriores autores en otros ámbitos. Es más, es muy probable que incluso se hayan producido con mayor intensidad, dado que el impacto de las políticas públicas de migración sobre la orientación del trabajo de las OSC -y ésta es una de nuestras principales hipótesis- puede que sea mucho mayor (Rincón, 2018). En realidad, la discusión sobre el papel de las OSC en el campo específico de las migraciones toma un carácter todavía más relevante, al situarse éstas en un campo crecientemente politizado, en la medida en que los Estados han incrementado su protagonismo y reforzado el control sobre los movimientos migratorios $y$, muy especialmente, en las fronteras que los migrantes tratan de traspasar. De modo que las OSC pro-migrantes han de posicionarse no sólo en el marco de las políticas públicas de acción social, sino también en el marco de las políticas migratorias y las políticas de seguridad, en contextos en los que -como ha ocurrido en España o en los Estados Unidos- las organizaciones han sido acusadas desde medios oficiales de promover la inmigración irregular o, incluso, de colaborar con las mafias que trasladan a las personas migrantes.

En este artículo tratamos de mostrar la posición que adoptan las OSC en las fronteras que separan México de los Estados Unidos y Marruecos de España, donde se ha creado un amplio y heterogéneo tejido asociativo en torno a estos espacios fronterizos (Fuentes-Lara, 2019). Con esta finalidad, el artículo se basa en los resultados de la investigación de campo llevada a cabo de octubre a diciembre de 2012 en la ciudad fronteriza de Tijuana y algunos puntos clave de la frontera norte de México con Estados Unidos ubicados en la región de Baja California y California, así como los obtenidos en el estudio realizado de julio a septiembre de 2014 en las ciudades autónomas de Ceuta y Melilla, además de otras localidades cercanas a la frontera sur de España con Marruecos y de territorio peninsular. La investigación incluye tanto una activa observación sobre el terreno, como entrevistas con los responsables de las organizaciones citadas en el artículo y otros informantes clave. 


\section{Las Organizaciones de la Sociedad Civil pro-migrantes y sus formas de acción}

Existe una gran diversidad de OSC que se involucran en la protección de los derechos humanos y el bienestar de los migrantes y refugiados. Las OSC emplean el capital social del que disponen para actuar ocupando distintos roles, ya sea reduciendo el coste humano de la migración (verbigracia las entidades de base confesional que proporcionan asistencia básica, alojamiento y otros servicios humanitarios), o para evitarla (con campañas de sensibilización llevadas a cabo por entidades que advierten sobre los peligros de migrar de manera irregular). Igualmente, pueden trabajar dentro de programas gubernamentales o llevar a cabo acciones sustentadas con fondos propios. Tal sería el caso de algunas asociaciones de migrantes, si bien existen otro tipo de organizaciones humanitarias e iniciativas ciudadanas que operan al margen de los Gobiernos sin percibir ningún tipo de financiación, e incluso oponiéndose a sus políticas migratorias (Gammeltoft-Hansen, Sørensen, 2013, p. 13-19).

De modo general, en función de la manera en la que se impliquen en las acciones de asistencia humanitaria y de reconocimiento, protección y defensa de los derechos humanos de migrantes y refugiados, podemos distinguir tres niveles de intervención (Tabla 1), que pueden darse de forma separada o simultáneamente: de primer grado (o asistencialismo básico), asistencialismo de segundo grado, y finalmente, de tercer grado o de incidencia (Rincón, 2016, p. 68-75; Rincón, 2018, p. 193-196; Moreno, Barajas, Figueroa, 2011, p. 3).

Las acciones de primer grado o de asistencialismo básico, son llevadas a cabo por organizaciones sin ánimo de lucro con fines altruistas, cuya única pretensión es proporcionar bienes o servicios primarios para cubrir las necesidades básicas de migrantes y refugiados que se encuentren en situación de vulnerabilidad. Entre ellos, se incluyen el alojamiento temporal, alimento, vestimenta, productos de higiene personal, atención sanitaria de primeros auxilios, e información referida a su situación legal o a los derechos que les asisten (Moreno et alii, 2011, p. 2,6-8; Rincón, 2018, p. 196-197). Es decir, son acciones impulsadas por los principios de solidaridad y cooperación que constituyen un primer acercamiento a la realidad (Gómez-Vargas, 2012, p. 16), desde una posición neutral o de no injerencia. Esto significa que, mediante su enfoque, no tratan de ahondar tanto en las causas estructurales que motivan a las personas a migrar de manera irregular, sino que las demandas son atendidas como respuesta a situaciones de carencia concretas (Oliva, 2001, p. 86; Moreno et alii, 2011, p. 3, 18-19). El problema del asistencialismo básico y la acción humanitaria es que pueden llevar a la excesiva victimización de la figura del migrante irregular y del refugiado, cuyas intervenciones apelen 
a la compasión, y no al cumplimiento de lo establecido por las leyes estatales o por el propio Derecho Internacional Humanitario, adoptando una posición paternalista (Andersson, 2014, p. 180; Ticktin, 2015, p. 291-296), que crea así "una figura sufriente y pasiva, recipiente de políticas de asistencia, antes que agente por derecho propio" (Schindel, 2017, p. 22).

En cuanto al asistencialismo de segundo grado (Rincón, 2016, p. 68; Rincón, 2018, p. 198), cubrirá las necesidades anteriores, pero habrá un mayor acercamiento a la realidad y una preocupación por las condiciones estructurales, acompañado todo ello de la interacción con actores locales y comunitarios (Gómez-Vargas, 2012, p. 16). En estos supuestos, los servicios prestados se amplían abarcando también la orientación jurídica, el apoyo en la elaboración de trámites administrativos, la mediación y acompañamiento, además de la asistencia psicológica o espiritual. Asimismo, primará la formación y los talleres de capacitación para promover la participación social y comunitaria, o para ayudar en la integración sociolaboral de las personas que son atendidas. Si bien es cierto que realizan acciones de promoción de los derechos humanos de los migrantes y refugiados con el objetivo de sensibilizar a la sociedad sobre la realidad de estas personas, no suelen involucrarlos en la defensa activa de sus derechos (Moreno et alii, 2011, p. 18-19).

Es de señalar que, en el asistencialismo de primer y segundo grado, es posible que las organizaciones estén financiadas tanto por programas públicos del Gobierno (Rincón, 2018, p. 204) en sus distintos niveles de administración, como por proyectos sustentados por organismos intergubernamentales y de cooperación al desarrollo, o a través de aportaciones y donativos procedentes de particulares y entidades privadas. Por otro lado, el capital humano del que disponen es en su mayor parte personal voluntario, prevaleciendo por encima del profesionalizado (Moreno et alii, 2011, p. 2).

Por último, las acciones de tercer grado se caracterizan por estar protagonizadas por grupos organizados que interactúan desde una posición reivindicativa con actores gubernamentales e intergubernamentales para la promoción de los derechos humanos de los migrantes y refugiados, quienes se involucran junto con voluntarios en las acciones de incidencia, que incluyen el apoyo de una determinada política o cabildeo, o la exigencia de la aplicación de la normativa estatal e internacional en la materia (Moreno et alii, 2011, p. 18-19; Gómez-Vargas, 2012, p. 16; Rincón, 2016, p. 53-54). En este nivel de intervención, los instrumentos fundamentales que se emplean son la elaboración de informes, la organización de eventos públicos y la celebración de acciones no violentas de denuncia de violación de derechos, cuyo fin es movilizar el apoyo a las causas pro migrantes, influenciar a la opinión pública y propiciar cambios políticos o sociales (Moreno et alii, 2011, p. 18-19). Finalmente, otra de las vías esenciales para coordinar sus acciones, es el 
trabajo en red mediante plataformas o a través de coaliciones de migrantes (Fox, 2010), que les permitirá no solo incrementar su impacto a nivel estatal, sino también contar con una mayor proyección internacional.

Tabla 1 - Cuadro con los tres niveles de intervención de las organizaciones de la sociedad civil en el ámbito de la ayuda humanitaria y defensa de los derechos de los migrantes y refugiados

\begin{tabular}{|c|c|c|c|}
\hline & $\begin{array}{c}\text { ASISTENCIALISMO BÁSICO O } \\
\text { DE } 1^{\text {er }} \text { GRADO }\end{array}$ & $\begin{array}{l}\text { ASISTENCIALISMO DE } 20 \\
\text { GRADO }\end{array}$ & $\begin{array}{l}\text { INTERVENCIÓN DE } 3^{\text {er }} \\
\text { GRADO O DE INCIDENCIA }\end{array}$ \\
\hline SERVICIOS & $\begin{array}{l}\text { - Alojamiento temporal } \\
\text { - Alimento } \\
\text { - Vestimenta } \\
\text { - Productos de higiene } \\
\text { personal } \\
\text { - Atención sanitaria } \\
\text { (primeros auxilios) } \\
\text { - Información básica sobre } \\
\text { derechos/situación legal }\end{array}$ & \begin{tabular}{l}
\multicolumn{1}{c}{$+{ }^{-}$} \\
- Orientación jurídica/ \\
trámites administrativos \\
- Mediación/ \\
acompañamiento \\
- Asistencia psicológica/ \\
espiritual
\end{tabular} & $\begin{array}{l}\text { No asistencialismo: } \\
\text { acciones de incidencia/ } \\
\text { cabildeo/ } \\
\text { Informes/denuncias }\end{array}$ \\
\hline PRINCIPIOS & $\begin{array}{l}\text { Solidaridad Cooperación } \\
\text { No injerencia } \\
\text { Neutralidad }\end{array}$ & $\begin{array}{l}\text { Solidaridad/Cooperación } \\
\text { >Acercamiento a la realidad } \\
\text { Interacción con actores locales/ } \\
\text { comunitarios }\end{array}$ & $\begin{array}{l}\text { Posición reivindicativa } \\
\text { frente a actores } \\
\text { gubernamentales e } \\
\text { intergubernamentales } \\
\text { No violencia }\end{array}$ \\
\hline $\begin{array}{l}\text { VISIÓN DE LAS } \\
\text { PERSONAS } \\
\text { ATENDIDAS }\end{array}$ & Sujetos pasivos & $\begin{array}{c}\text { Se busca capacitarlas/ } \\
\text { participación social/ } \\
\text { comunitaria } \\
\text { Integración sociolaboral } \\
\text { No defensores activos de sus } \\
\text { propios derechos }\end{array}$ & $\begin{array}{l}\text { Sujetos activos. } \\
\text { Actores en la defensa de } \\
\text { sus derechos humanos. }\end{array}$ \\
\hline $\begin{array}{l}\text { FINANCIACIÓN/ } \\
\text { ORGANIZACIÓN }\end{array}$ & \multicolumn{2}{|c|}{$\begin{array}{c}\text { Programas públicos de Gobierno } \\
\text { Proyectos de organismos Intergubernamentales/cooperación } \\
\text { al desarrollo } \\
\text { Donativos de particulares/entidades privadas }\end{array}$} & $\begin{array}{l}\text { Trabajo en Red/ } \\
\text { Plataformas } \\
\text { Coaliciones de migrantes }\end{array}$ \\
\hline PERSONAL & \multicolumn{2}{|c|}{$\begin{aligned} & >\text { Voluntarios } \\
< & \text { Profesionales }\end{aligned}$} & Voluntariado \\
\hline
\end{tabular}

Fuente: Elaboración propia.

\section{La acción de las OSC en la frontera entre Estados Unidos y México}

En la frontera de Estados Unidos-México, el recrudecimiento de la política migratoria estadounidense de control interno, dio lugar a que durante el periodo que va de 2003 a 2015, hubiera una mayor presencia de mexicanos que eran deportados desde los Estados Unidos, quienes quedaban varados en los principales puntos de repatriación de México, como sucedió en las ciudades fronterizas de Tijuana y Mexicali. Este aspecto se hizo especialmente visible con el Programa Comunidades Seguras activado desde 2008, dado que las deportaciones masivas de mexicanos, generaron un grave problema humanitario para el Gobierno Federal de este país, cuyas políticas en la materia fueron insuficientes. Organizaciones como la Comisión Binacional de Derechos 
Humanos $(\mathrm{CBDH})$ denunciaron que los migrantes mexicanos deportados se encontraban con diversas trabas administrativas, y no se activaban dispositivos de emergencia que evitaran que las personas acabaran en condición de calle, o cayeran en el consumo de drogas y alcohol, debido a la inexistencia en ese momento de un programa de atención integral del fenómeno migratorio como país de origen, tránsito, destino y retorno.

Ante la falta de un mayor número de albergues y comedores gratuitos, además de programas de inserción sociolaboral efectivos que favorecieran la integración de los migrantes deportados y evitaran su exclusión social, durante 2012 adquirió gran relevancia la labor asistencialista que desarrolla en Tijuana la Congregación de Misioneros y Misioneras de San Carlos Borromeo (también conocidos como Scalabrinianos/Scalabrinianas) dentro del Programa de Repatriación Humana (PRH). Este programa es implementado por el Gobierno Federal de México en coordinación con los albergues de la Casa del Migrante y de la Casa Madre Asunta, los cuales forman parte de la Coalición Pro Defensa del Migrante (COALIPRO), cuyo fin es colaborar con los funcionarios del Instituto Nacional de Migración del Gobierno de México (INM) para que las repatriaciones de ciudadanos mexicanos desde Estados Unidos se hagan de manera ordenada, legal y segura, al tiempo que han procurado fomentar su reinserción en México. En ellos prestaban respectivamente servicios asistenciales a los hombres y mujeres que eran deportados por la garita de "El Chaparral", y su nivel de intervención puede ser calificado de primer y segundo grado, puesto que el área de trabajo social de estas entidades no solo se centraba en cubrir las necesidades inmediatas que las personas migrantes en tránsito y deportadas presentaban al llegar a las ciudades fronterizas, sino también en detectar casos de malos tratos o irregularidades en el proceso de deportación.

La asistencia humanitaria que proporcionaba el albergue de la Casa del Migrante, que contaba con una capacidad de hasta $180^{2}$ personas, era totalmente gratuita, y comprendía los servicios siguientes: alojamiento, baño con agua caliente, alimentación, primeros auxilios, ropa, calzado, mantas, y enseres para el aseo personal (cepillo de dientes, jabón, etc.). Pero pasado el plazo de permanencia de 12 días, tenían que abandonarlo, si bien a día de hoy las personas migrantes que son atendidas, pueden permanecer en el mismo hasta por 45 días. E incluso esta organización ha implementado un programa a través del cual, cuando abandonen el albergue y renten una vivienda, puedan regresar durante 6 meses para comer, recibir atención sanitaria, y acceder a los servicios que ofrece el centro. En lo que respecta a la Casa Madre Asunta, técnicamente tenían una capacidad para 45 personas,

\footnotetext{
2 El albergue contaba en 2019 con 150 camas.
} 
quienes podían permanecer 15 días, aunque a veces se prolongaba su estancia si estaban siguiendo algún proceso judicial.

Es de señalar que la política migratoria de detención y deportación de migrantes, fue aplicada en algunas ocasiones con extraordinaria agresividad y brutalidad. Así pues, era habitual que las personas migrantes repatriadas, relataran haber sido víctimas de episodios violentos durante su proceso de detención, lo que les producía un daño físico y psicológico difícil de reparar, especialmente una vez habían sido deportadas. De ahí la relevancia del servicio de orientación jurídica de estos albergues, desde donde pretendían que tomaran conciencia de que eran titulares de derechos, y que, por esta razón, no debían asumir o normalizar los excesos cometidos por parte de las autoridades migratorias estadounidenses, ya que existían mecanismos legales que los amparaban y que podían ser activados por organizaciones pro migrantes en los Estados Unidos. Igualmente, para el Centro Madre Asunta, la asistencia legal se convertía en un apoyo vital para aquellas madres que trataban de iniciar un proceso de reunificación familiar, cuando el gobierno estadounidense había asumido la custodia de sus hijos.

En lo que respecta a otros servicios, como la atención psicológica, eran prestados de una manera precaria, dado que tenían que recurrir a voluntarios o estudiantes universitarios, sin poder ofrecer un servicio institucionalizado dentro de la organización. Del mismo modo, no era posible realizar itinerarios de inserción sociolaboral. Aunque estos aspectos han sido mejorados en la actualidad mediante la ampliación de los Departamentos de Atención Directa a Migrantes dentro de la Casa del Migrante, o con la bolsa de trabajo del Centro Madre Asunta. Luego, la llegada masiva de refugiados a la ciudad de Tijuana, ha hecho que hayan tenido que intensificar sus esfuerzos para la movilización de efectivos de apoyo, más aún desde que el gobierno de López Obrador ha eliminado la partida presupuestaria que era destinada al programa social de apoyo de los albergues situados a lo largo de la frontera (Alvarado, 11 de diciembre de 2019).

Otro de los rasgos que destaca de estos albergues, es su extraordinaria disciplina, que contrasta con la forma improvisada de trabajar del "Hotel Migrante" de Mexicali, gestionado por Ángeles sin Fronteras, una entidad cuyo fundador Sergio Tamai estaba vinculado a la fe mormona. Este proyecto había surgido de la solidaridad espontánea de un grupo de personas, cuyos miembros, más contestatarios, intervenían en el ámbito asistencial al margen de los programas gubernamentales mexicanos, lo cual ocasionaba alguna confrontación con las instituciones oficiales, así como ciertas reticencias por parte de otras organizaciones de la sociedad civil mexicanas más implicadas con el Gobierno, que ponían en duda la efectividad real de sus acciones. 
Uno de los aspectos que más controversia ocasionaba (al margen de la forma en la que se ocupó ${ }^{3}$ el edificio), es que en este centro convivían migrantes deportados con aquellos que practicaban el coyotaje, o sufrían algún tipo de dependencia a las drogas y el alcohol. Algo que era impensable en la Casa del Migrante de Tijuana, ya que las normas del centro contemplaban estas prácticas como impedimento de entrada o como posible causa de expulsión del albergue. Por otro lado, si bien es cierto que su nivel de intervención se limitaba al asistencialismo básico, y que contaba con un trabajo algo estructurado por áreas de intervención, no disponían de medios para la elaboración de informes, ni para hacer seguimientos o itinerarios de inserción sociolaboral.

Sin embargo, pretendían que los migrantes deportados trataran de ser autónomos y asumieran protagonismo como defensores de la causa migrante, aunque sus métodos fueran cuestionables. De hecho, pese a que acabaron percibiendo fondos públicos (fruto de las presiones que ejercían mediante las protestas en las calles), la falta de recursos era suplida con el trabajo voluntario prestado por los propios migrantes dentro del Hotel (que incluía la recaudación de fondos a pie de calle), o con la compra de alimentos en uno de los negocios que pertenecía a su fundador. A esto se le sumaban las deficiencias de la infraestructura del edificio, que dificultaban enormemente su mantenimiento, de modo que se vieron obligados a cerrar sus puertas en varias ocasiones por impagos de la renta y la electricidad, llegando a no poder garantizar la alimentación de sus inquilinos. Un problema que persiste en la actualidad. Asimismo, como forma de ampliar su red, esta entidad se apoyaba en su homóloga estadounidense "Border Angels", la cual desarrollaba (y desarrolla) varios programas binacionales que tienen carácter asistencialista, como llevar depósitos de agua al desierto o dar asesoría legal gratuita a migrantes en San Diego y en el Parque de la Amistad del muro fronterizo con Tijuana. De igual modo, realizan una labor divulgativa y formativa para la defensa de los derechos humanos y civiles de las personas migrantes en tránsito, deportadas y solicitantes de asilo. Incluso han puesto en marcha pequeñas iniciativas en la ciudad de Tijuana, con el fin de proporcionarles alguna oportunidad laboral.

\footnotetext{
3 Este hotel, que abrió sus puertas a los indigentes el 15 de enero de 2010, está situado en un antiguo edificio abandonado, que dista de una cuadra del puerto de entrada fronterizo Caléxico, CA - Mexicali. En él se ofrece hospedaje, comida, ropa y protección a migrantes. El Hotel fue construido como lugar de paso en el año 1935 junto a la primera aduana de Mexicali, y llevaba durante los años 40 y 50 el nombre de "Hotel Santa Clara". Posteriormente pasó a ser conocido como el "Hotel Centenario", un lujoso negocio que acabó siendo desatendido por sus administradores en el año 2000 (Marosi, 26 de mayo de 2012). Es por ello que se desconoce si los componentes de la organización cívica pidieron alguna licencia o si hicieron algún trámite, hasta el punto de que hay quienes sostienen que fue literalmente ocupado, mientras que otras fuentes señalan que pagaban una renta de $1.000 \$$ por el alquiler del mismo.
} 
Por último, pese a que las entidades vinculadas a la COALIPRO colaboraban con el Gobierno Federal, adoptaban una posición más reivindicativa al llevar a cabo acciones de incidencia. En este sentido, colaboraban con organismos de investigación (como El Colef), en la elaboración de informes a partir de los testimonios recogidos por el área de Trabajo Social. Esta misma táctica era empleada a través de la Revista "Migrantes", mediante la cual los Scalabrinianos difunden su labor, con la finalidad de denunciar las injusticias, la violencia y las vulneraciones sistemáticas de los derechos humanos de las personas que se ven obligadas a migrar de manera irregular. Simultáneamente, su lucha social se veía reforzada a través de la celebración de acciones no violentas en fechas clave, como las "Posadas Migrantes". En ellas, involucraban a otras organizaciones de San Diego que formaban parte de la Iglesia Protestante y de centros universitarios de la ciudad, al tiempo que convertían en protagonistas a las personas deportadas. Así pues, mediante la exposición pública de sus experiencias migratorias, pasaban de ser meros receptores de la ayuda y asistencia humanitaria, a promotores activos en la defensa de sus derechos humanos. Mas sus actos de protesta suelen estar impregnados de cierto simbolismo cristiano, que enfatizan la imagen sufriente de las personas migrantes, refugiadas y deportadas que son víctimas de las políticas de control migratorio, en las que se recurre a metáforas que relacionan la historia de la huida de José de Nazaret y el nacimiento de Jesús, con la dura travesía de su tránsito migratorio. En ese sentido, las otras entidades que actúan al margen de los gobiernos, como los Border Angels, participan, al igual que hicieran con las "Marchas Migrantes, en los "Vía Crucis" de refugiados centroamericanos, portando cruces como muestra del paralelismo del padecimiento de aquellos que migran forzosamente, con el calvario de Jesús antes de su muerte.

\section{La acción de las OSC en la frontera entre España y Marruecos}

En el caso de la frontera España-Marruecos ésta cuenta con la particularidad de situarse en torno a las ciudades enclaves de Ceuta y Melilla, alrededor de las cuales se han erigido sendas vallas que han venido elevándose y reforzándose de forma continuada, en paralelo con el endurecimiento de las políticas migratorias de España y la Unión Europea en su única frontera terrestre con África.

Allí, la Archidiócesis de Rabat y Tánger actúa como uno de los principales actores de la sociedad civil, compartiendo la misma línea de trabajo que las organizaciones Scalabrinianas, ya que desarrollan varios proyectos dentro de las zonas pastorales de Tánger y Nador en Marruecos. La Archidiócesis actúa a través de sus Delegaciones Diocesanas en los puntos principales de espera, que se ubican en las barriadas más pobres de las urbes y en las zonas de 
los campamentos forestales de Benyunes y el Gurugú, cercanos a las vallas de las ciudades fronterizas de Ceuta y Melilla. El proyecto de 2014 que era ejecutado en Tánger (conocida por ser uno de los lugares de salida de las pateras hacia aguas del Estrecho), consistía en un programa de atención directa e integral financiado con fondos europeos, cuyo fin era evitar que los migrantes subsaharianos cayeran en situación de exclusión social, mientras promovían la defensa de sus derechos humanos, si bien tuvieron que ampliar su campo de acción ante la llegada masiva de las familias de refugiados sirios. Esta actividad puede calificarse de asistencialismo de primer y segundo grado, ya que las ayudas sociales que proporcionaban, estaban dirigidas a solventar necesidades básicas, como la entrega de ropa, mantas, kits escolares para niños, o para cubrir gastos derivados de trámites administrativos, transporte, vivienda, etc.

En lo que respecta a la intervención de segundo grado, hay que destacar que se centraban en mayor medida en la atención de los grupos más vulnerables, esto es, las mujeres y sus hijos. Para ello, realizaban acompañamientos sociosanitarios en los hospitales mediante mediadores, poniendo especial énfasis en la asistencia a las embarazadas, igual que hacían en los bosques de Benyunes. En cuanto a la atención psicológica, contaban con un servicio altamente cualificado, que incluía tratamientos individualizados, así como terapias grupales, en las que atendían por igual a los niños. Otro de los puntos fuertes de este programa, era la educación y la formación para promover el autoempleo mediante la artesanía.

El enfoque de la Delegación de Migraciones en los campamentos del Gurugú en Nador, era principalmente asistencialista. Allí, actuaban a través de un equipo compuesto por agentes de proximidad, dentro de un proyecto financiado por la Cooperación Suiza y la OIM. Sin embargo, su campo de acción se veía enormemente limitado por las extremas condiciones de vida de los bosques y la vigilancia que se ejercía sobre esa área, pues sus actividades eran supervisadas continuamente por las autoridades marroquíes ${ }^{4}$. A diferencia de Tánger, donde resultaba más fácil hacer seguimientos, esta labor se complicaba en este área. Allí trabajaban con una población flotante que fluctuaba constantemente, debido a que algunos de los habitantes de los asentamientos se marchaban a otros lugares o saltaban la valla, y las cifras que habían sido incluidas en los mapeos de la zona, simplemente servían de referencia. Es por ello que únicamente podían prestar atención directa sanitaria, mediar en los hospitales y distribuir de vez en cuando material de

\footnotetext{
4 Tanto es así, que el 11 de enero de 2016, cuando el padre Esteban SJ trataba de entrar a Nador por la frontera de Melilla, se le denegó la entrada al país y se le requisó su permiso de residencia marroquí, bajo la triple acusación de ejercer el proselitismo religioso, de ir en contra de la unidad territorial de Marruecos y de ser un espía del Servicio Secreto Español (CNI).
} 
ayuda humanitaria, momentos que aprovechaban para socializar con ellos e informarles sobre el proceso de regularización de migrantes irregulares que se había iniciado en Marruecos. Obviamente, las actividades formativas estaban totalmente excluidas de este proyecto.

En cuanto a los programas de primera acogida que el gobierno español ejecuta mediante los Centros de Estancia Temporal de Inmigrantes (CETI) de Ceuta y Melilla, las ONG que trabajan dentro de estos espacios, inevitablemente ven ligados sus objetivos humanitarios con el cumplimiento de las exigencias securitarias de control de fronteras, dado que están dirigidos por altos funcionarios de las Fuerzas Armadas y Cuerpos de Seguridad del Estado, los cuales se hallan en situación de excedencia voluntaria. Este aspecto es especialmente visible en el caso de Cruz Roja, que no solo percibe subvenciones estatales para ofrecer servicios humanitarios dentro de los CETI, sino que amplía su campo de acción para que las aprehensiones a pie de valla o en las costas, se hagan garantizando la vida e integridad física de las personas que tratan de vulnerar el perímetro fronterizo. Es decir, su posición de neutralidad, le lleva a un nivel de intervención calificable como asistencialismo de primer grado, desde donde no se cuestionan los efectos nocivos de las actuaciones policiales de vigilancia fronteriza.

Cuando los migrantes son enviados a los CETI, la intervención de Cruz Roja pasa a ser de segundo grado. En estos lugares, la Administración Pública colabora con las ONG y demás entidades, para prestar servicios básicos de comida, alojamiento, asesoramiento jurídico y asistencia psicológica, haciendo especial incidencia en la formación y preparación de esas personas, antes de ser enviados a la Península. Así pues, los trabajadores y voluntarios actúan bajo supervisión de los funcionarios gubernamentales, siguiendo un estricto protocolo de atención, a través del cual, los residentes, previamente identificados con una tarjeta de control, han de pasar necesariamente por todas las áreas de intervención antes de poder acceder al resto de los servicios. Todos ellos son ofrecidos de manera profesionalizada, esto es, por técnicos especialistas, si bien cuentan también con en el apoyo de voluntarios, especialmente para el desarrollo de actividades educativas y de ocio.

En cuanto a las intervenciones de tercer grado, conviene subrayar que el hecho de que las organizaciones españolas percibieran financiación gubernamental e internacional dentro del marco de programas europeos, de cooperación al desarrollo o de la OIM, les obligaba en cierto modo a mantener un discurso de no confrontación con las instituciones, mediante el que defendían la vía del encuentro entre los organismos sociales y las fuerzas del orden. Ahora bien, sus representantes, el Arzobispo emérito Agrelo ${ }^{5}$ y el

5 El 24 de mayo de 2019 el Papa Francisco aceptó la renuncia al cargo de Arzobispo de Tánger, que fue presentada en junio de 2017 por motivos de edad. 
Padre Esteban Velázquez SJ, llevaban a cabo denuncias y protestas públicas para visibilizar las condiciones en las que se encontraban los migrantes irregulares dentro de Marruecos, ya fuera valiéndose de las redes sociales, los medios de comunicación o mediante la celebración de Jornadas, lo que les llevaba a un nivel de intervención de tercer grado. Claro está, no involucraban activamente en estas acciones a los propios migrantes, porque su situación de "clandestinos" no lo permitía. Otra de las herramientas de incidencia que utilizaban, era el fortalecimiento de las redes de trabajo, colaborando tanto con asociaciones locales, regionales y nacionales marroquíes (a las que les derivaban algunos casos) como con organizaciones internacionales.

En lo que respecta a Cruz Roja se limita a la elaboración de informes y memorias, que den cuenta de la actividad que han desarrollado y que sirvan para sensibilizar sobre la realidad migratoria de estas personas. Aunque existen otras entidades dentro de los CETI que ejercen un papel más contestatario, como la Comisión Española de Ayuda al Refugiado (CEAR), porque, aunque también es subvencionada por el gobierno español para dar orientación jurídica y asistencia legal en materia de asilo y refugio, forma parte de la Red euroafricana Migreurop, a través de la cual defienden a las personas migrantes y refugiadas frente a los abusos y maltratos que sufren en las fronteras. Es por este motivo que se han llegado a personar en algunos procesos judiciales como acusación particular junto con otras organizaciones, en los que han buscado depurar responsabilidades y que se visibilice lo que sucede en las vallas, para que cese la violencia en ellas y se deje de emplear métodos disuasorios tan agresivos como las concertinas. Otras integrantes de esta red, entre las que se encuentran Andalucía Acoge, la Asociación Pro Derechos Humanos de Andalucía (APDHA) y la asociación Elín (representada por la Hermana Paula Domingo, Carmelita de la Caridad Vedruna), también emiten informes y elevan manifiestos, donde denuncian las vulneraciones de derechos humanos ante organismos internacionales y el Parlamento Europeo (a las que se suman también el Servicio Jesuita al Migrante, SJM), exigiendo la implantación de vías legales y seguras.

En una línea más reivindicativa y de trabajo colaborativo se encuentran varias entidades que mantienen una estrecha relación con la APDHA. Éstas son la asociación Pateras de la Vida (la cual forma parte de la Red Chabaka de asociaciones del norte de Marruecos), Pedagogía Ciudadana, Digmun y Elín, que a parte de la labor de sensibilización que realizan en Larache y en Ceuta, participan anualmente en las "Marchas de la Dignidad" en honor a las quince personas migrantes de origen subsahariano que fallecieron ahogadas cuando trataban de acceder a nado por el espigón de la frontera del Tarajal, a consecuencia de las acciones disuasorias de la Guardia Civil española. Estos actos pacíficos impulsados desde sus inicios por la asociación 
Elín, son coordinados ahora con una gran red de organizaciones españolas y europeas pro-migrantes. En estas marchas, se realizan toda clase de actos simbólicos en recuerdo de las víctimas, como la colocación de placas con sus nombres, cánticos o la lectura de manifiestos. Pero lo más relevante, es que son encabezadas desde el CETI de la ciudad por los propios migrantes subsaharianos, quienes portan pancartas y lanzan proclamas al unísono de los manifestantes, momento en el que tienen la oportunidad de erigirse como sujetos activos en la defensa de sus derechos humanos, ya que además asisten a las mesas redondas que se celebran junto con los representantes de las ONG.

Dicha labor se extiende hasta territorio peninsular mediante los "Círculos del Silencio" que se convocan mensualmente, en los que se apela la conciencia de los legisladores, para que modifiquen aquellas leyes migratorias que atentan contra la dignidad de las personas. A esto se le agregan en Valencia las "Vigilias" pascuales en la puerta del Centro de Internamiento de Extranjeros de Zapadores (CIE) y las marchas por Navidad en el "Día del Internacional del Migrante" organizadas por la plataforma CIES No, que aglutina a distintos colectivos y asociaciones de base migrante. Su fin es reclamar la clausura definitiva de estos centros, que son definidos por sus integrantes como espacios de represión y sufrimiento, al tiempo que piden que se acabe con la criminalización de los migrantes, las redadas racistas y las deportaciones masivas. Por otra parte, también hacen acompañamientos a los internos y emiten informes donde denuncian casos de malos tratos e irregularidades en los procedimientos de detención y deportación.

\section{Discusión de los resultados}

El derecho a recibir y a brindar asistencia humanitaria, constituye un principio humanitario fundamental que asiste a todo ciudadano en todo país, y que es necesario para hacer efectivos otros derechos humanos fundamentales, entre los que se incluye el derecho a la vida, la alimentación, la vestimenta y la vivienda. No obstante, Scott D. Watson (2011, p. 6) reconceptualizó la idea de humanitarismo ubicándola dentro de la lógica securitaria del Estado nación, al considerarlo un sector más de la seguridad, que concibe como amenazas existenciales para la vida y la dignidad humana, aquellas que generen escenarios de vulnerabilidad, pobreza e injusticias, o que provoquen la pérdida de vidas a gran escala. En atención a lo cual, la seguridad humanitaria se verá garantizada a través de medidas de emergencia dirigidas a prestar una ayuda imparcial y neutral de socorro, o mediante intervenciones militares, que han de ser planificadas de manera coordinada, implicando conjuntamente a los Gobiernos, las organizaciones multilaterales y las ONG (Benavides, 2009, p. 119). 
Uno de los aspectos más problemáticos que plantea la ayuda humanitaria en el campo del control migratorio, proviene del hecho de que los Gobiernos subsidien a las ONG para garantizar que la gestión securitaria de las fronteras se haga respetando los derechos humanos (Varela, 2015; O'Hagan, 2013, p. 131, citado por Ruíz-Giménez, 2017, p. 180). Esto plantea una relación de enorme complejidad entre estas organizaciones y los Estados donantes, ya que las fuerzas de policía y la acción humanitaria convergen en albergues o centros de estancia temporal, o incluso dentro de los centros de detención e internamiento, propiciando un escenario de emergencia perpetuo (Agier, 2011; Treviño, 2016).

Tanto en Estados Unidos como en España los Estados han ido derivando la gestión migratoria a terceros o a actores privados, que asumen la función de asistir a los migrantes en espacios controlados, ahondando así en la instrumentalización de la ayuda humanitaria que se pone al servicio de la seguridad (Ruíz-Giménez, 2017, p. 177-180; Hernández-León, 2013, p. 32). Entre ellos, se encuentran los funcionarios de instituciones que ejecutan programas de primera acogida, organizaciones humanitarias y defensoras de derechos humanos religiosas y laicas que atienden a migrantes irregulares en tránsito o cuando son deportados, y las redes de migrantes. Así que se ha generado toda una "industria del rescate" (Agustín, 2007) conformada por actores estatales y no estatales, que actúan tratando de que la migración internacional sea más ordenada y humanitaria, pero que acaban viéndose involucrados en las funciones de control (Gammeltoft-Hansen, Sørensen, 2013, p. 13-15).

En su estudio de las organizaciones que prestan servicios a la migración en Carolina del Sur (USA) Roth, Park y Grace (2018) muestran cómo éstas intermedian entre los inmigrantes y un contexto político cada vez más restrictivo, y cómo no todas ellas se sitúan en la línea de la defensoría de derechos, en función de factores como el tamaño de la organización, las fuentes de financiación, la disposición de los líderes y su predisposición a colaborar (Roth, Park, Grace, 2018, p. 697).

En la frontera de México-Estados Unidos, las entidades religiosas han suplido el papel protector correspondiente al Estado dentro del ámbito de su jurisdicción, en buena medida ante la situación de desamparo que vivían las personas deportadas. Asimismo, las acciones de incidencia política de las organizaciones pro-migrantes mexicanas y estadounidenses en sus distintos niveles de intervención, han sido de gran relevancia para visibilizar las vulneraciones de derechos humanos que sufren estas personas en los procesos de detención y deportación. Sirva de ejemplo cuando CHIRLA logró en 2013 que el Sheriff del Condado de Los Ángeles limitara su participación en el Programa Comunidades Seguras, comprometiéndose a no enviar huellas 
dactilares al ICE en aquellos supuestos en los que hubieran cometido un delito menor. Pero también han servido para combatir la discriminación que han sufrido (y sufren) los deportados mexicanos cuando llegan a los puntos de repatriación como Tijuana, pues gracias a la presión ejercida, se consiguió un mayor grado de implicación del Gobierno Federal, quien finalmente se vio obligado a activar programas que trataran de favorecer su integración sociolaboral.

En la frontera de España-Marruecos, el objetivo de las organizaciones ha sido crear una gran red internacional de entidades que aúne sus fuerzas en plataformas compuestas por organizaciones pro-migrantes, ONG y asociaciones de base migrante, para lograr un cambio en la política migratoria de gestión fronteriza y de control interno, priorizando el aspecto humanitario y la solidaridad por encima de los requerimientos de la seguridad nacional, tanto en el ámbito español como en el europeo. En este mismo sentido, también es relevante el papel que ha ejercido la Iglesia Católica a través de sus distintas órdenes religiosas, cuyas actividades han buscado el diálogo institucional para la consecución de sus fines humanitarios. No obstante, como han señalado algunas investigaciones, siempre hay grupos de población que quedan al margen de la acción de las OSC, como el caso de las porteadoras marroquíes (Fuentes-Lara, 2019).

En ambos casos, las organizaciones de base confesional han actuado más allá del asistencialismo desde un enfoque de derechos humanos, considerando a las personas migrantes en tránsito, deportadas y refugiadas, sujetos titulares de derechos, lo que les ha permitido superar la visión caritativa de éstas como seres vulnerables. Además, las entidades de ambas latitudes, combaten la criminalización y el estigma que arrastran cuando son interceptadas en las fronteras o cuando son detenidas y deportadas, o se implican junto con los migrantes de manera activa en la promoción y convocatoria de acciones no violentas de denuncia pública mediante movilizaciones masivas y de sensibilización. De esta manera, favorecen que su voz sea oída más allá de los albergues y centros de estancia donde son atendidos. Pero también se valen de instrumentos como son la elaboración de informes o manifiestos, que hacen de altavoz de sus demandas y reivindicaciones, y que pueden servir como forma de presión para que los gobiernos destinen una mayor dotación de recursos a programas que vayan más allá de las intervenciones puntuales en situaciones de crisis o emergencia humanitaria, o para evitar que se incumpla la normativa nacional e internacional protectora de sus derechos humanos.

\section{Conclusiones}

En el pasado el trabajo de las OSC se ha visto bien como complementario, bien como sustitutivo o bien como alternativo al papel del Estado, generando 
una serie de debates que han tenido sobre todo lugar en las sociedades del Norte y, cuando hablamos específicamente del ámbito de la migración, en los lugares que han sido protagonistas en la recepción y asentamiento de los migrantes. En la actualidad, esta triple diferenciación se habría ido desdibujando -en especial en esos espacios de frontera, de tránsito o simplemente no lugares-, donde en contextos de alta complejidad muchas de las OSC han ido readaptando sus posicionamientos en la medida en que los escenarios han ido alterándose y las políticas han venido también modificándose. Así, el medio en el que operan las OSC se encuentra atravesado por las políticas de las instituciones locales, las políticas nacionales de los Estados a un lado y otro de la frontera, y, en el caso de la frontera hispano-marroquí, también por las políticas supranacionales de la Unión Europea, lo que dificulta notablemente su acción.

Las características de los contextos en que las organizaciones desarrollan su actividad resultan fundamentales para entender los posicionamientos de las mismas en términos de humanitarismo y la defensa de derechos o advocacy (Almog-Bar, Schmid, 2014), o al situar sus acciones entre el asistencialismo básico, el asistencialismo de segundo grado y la incidencia política. Pese a que muchas de las OSC que actúan en ambos espacios fronterizos siguen desarrollando labores que podemos calificar como de asistencialismo básico, la gravedad de las situaciones que se viven y la constante vulneración de derechos de los migrantes, coloca también las mismas -tanto las de carácter religioso como no religioso- en la necesidad de desplegar acciones de incidencia política.

Paradójicamente, la posibilidad de acción crítica de las OSC se presenta mucho más dificultosa respecto a las políticas asistenciales y de integración de los inmigrantes que en el de las políticas de control de los flujos, en tanto que en este último terreno la dependencia financiera de las OSC es mucho menor que en el ámbito de la integración social. Ello ocurre sobre todo entre las OSC que trabajan no tanto en contextos de excepcionalidad -como son los espacios de frontera- como en lugares de asentamiento ya alejados de la emergencia humanitaria (las numerosas OSC pro-migrantes que trabajan dentro del territorio de los Estados Unidos o de España, alejadas de las fronteras en crisis permanente). Tanto las labores de defensoría (advocacy) como de incidencia política de las OSC suelen ser mucho más activas frente a las políticas de control desplegadas en las mismas fronteras, y allí sus acciones suelen polarizarse en mayor medida entre el asistencialismo más básico de tipo humanitario y las reivindicaciones y las denuncias en la actuación de los Estados.

Por tanto, las OSC se han de adaptar a un contexto político muy cambiante, al tiempo que la capacidad de incidir sobre el mismo resulta enormemente limitada, con el riesgo añadido de que con su labor humanitaria el foco de 
atención pública se desvíe hacia las personas migrantes como víctimas, en lugar de hacia las políticas que deshumanizan crecientemente las fronteras.

\section{Bibliografía}

AGIER, Michel. Managing the Undesirables: Refugee Camps and Humanitarian Government. Cambridge: Polity Press, 2011.

AGUSTíN, Laura. Sex at the Margins: Migration, Labour Markets and the Rescue Industry. London: Zed Books, 2007.

ALMOG-BAR, Michael; SCHMID, Hillel. Advocacy Activities of Nonprofit Human Service Organizations: A Critical Review. Nonprofit and Voluntary Sector Quarterly, v. 43, n. 1, p. 11-35, 2014.

ANDERSSON, Ruben. Illegality, Inc.: Clandestine migration and the business of bordering Europe. Oakland: Ed. University of California Press, 2014.

ALVARADO, Isaias. Migrantes venden pupusas para mantener un albergue en Tijuana. Univisión, 11 de diciembre de 2019. Disponible en: <https://xurl.es/ csp6n>. Acceso en: 13.12.2012.

BENAVIDES, Lourdes. El régimen internacional del "nuevo humanitarismo". Relaciones Internacionales, n. 12, GERI-UAM, p. 107-124, octubre 2009.

COMISIÓN ESPAÑOLA DE AYUDA AL REFUGIADO (CEAR). Las organizaciones de Migreurop en España piden la intervención diplomática española tras la expulsión de Esteban Velázquez por parte de Marruecos, 28 de enero de 2016. Disponible en: <https://bit.ly/2TCxS5W>. Acceso en: 20.03.2019.

FOX, Jonathan; GOIS, William. La sociedad civil migrante: diez tesis para el debate. Migración y desarrollo, v. 8, n. 15, p. 81-128, 2010.

FUENTES-LARA, M. Cristina. El papel del tercer sector de la región fronteriza hispano-marroquí en la situación de las mujeres porteadoras en Ceuta. Revista de Estudios Internacionales Mediterráneos, v. 26, p. 144-159, 2019.

GÓMEZ-VARGAS, Irazú. Ayuda Humanitaria: ¿Construcción de Ciudadanía? Seminario Permanente sobre Migración Internacional. En Sesión 7ạ. Ciclo 2012. Escenarios de violencia de las migraciones internacionales: actores y respuestas. El Colef, 2012.

HERNÁNDEZ-LEÓN, Rubén. Conceptualizing the migration Industry. In: GAMMELTOFT-HANSEN, Thomas; SØRENSEN, Ninna Nyberg (eds.). The Migration Industry and the Commercialization of International Migration. Nueva York: Ed. Routledge, 2013, p. 24-44.

HIRSCHMAN, Albert O. Exit, Voice, and Loyalty: Responses to Decline in Firms, Organizations and States. Cambridge: Harvard University Press, 1970.

MAROSI, Richard. In Mexicali, a haven for broken lives. Los Ángeles Time, 26 de mayo de 2012. Disponible en: <https://lat.ms/2Vs9plN>. Acceso en: 02.01.2013.

MCMULLIN, Caitlin; SKELCHER, Chris. The impact of societal-level institutional logics on hybridity: Evidence from nonprofit organizations in England and France. Voluntas, v. 29, p. 911-924, 2018. 
MINKOFF, Debra C. From service provision to institutional advocacy: the shifting legitimacy of organisational forms. Social Forces, v. 72, n. 4, p. 943-969, 1994.

MINKOFF, Debra C. The Emergence of Hybrid Organizational Forms: Combining Identity-Based Service Provision and Political Action. Nonprofit and Voluntary Sector Quarterly, v. 31, n. 3, p. 377-401, 2002.

MORENO, José A.; BARAJAS, Margarita; FIGUEROA, Leticia. Estructura, funcionamiento y modelos de atención de las organizaciones civiles de apoyo a los migrantes en la frontera norte de México. IV Congreso de la Red Internacional de Migración y Desarrollo. Crisis Global y Estrategias Migratorias: hacia la redefinición de las políticas de movilidad, 18, 19 y 20 de Mayo del 2011. Quito Ecuador: Flacso, 2011.

OLIVA, Andrea. Análisis de la práctica profesional de los trabajadores sociales en el ámbito estatal (Maestría en Servicio Social). Pontificia Universidade Catolica São Paulo, bajo la orientación de la Profa - Dra . Dilsea Bonetti. Buenos Aires, 2001.

RINCÓN, Elvira. La sociedad civil organizada responde al impacto de políticas públicas: Las políticas sociales como factor causal del rol asistencialista de organizaciones de la sociedad civil (OSC) pro migrantes en Tijuana. Gestión y política pública, v. 27, n. 1, p. 181-209, 2018.

RINCÓN, Elvira. La cultura política como factor causal del predominio asistencialista en la atención a grupos vulnerables: las Organizaciones de la Sociedad Civil pro migrantes en Tijuana. Revista Mexicana de Análisis Político y Administración Pública, v. V, n. 2, p. 53-78, julio-diciembre 2016.

ROTH, Benjamin; PARK, Seo Yeon; GRACE, Breanne. Advocating for Structural Change? Exploring the Advocacy Activities of Immigrant-Serving Organizations in an Unwelcoming Policy Context. Advances in Social Work, v. 18, n. 3, p. 682703, 2018.

RUÍZ-GIMÉNEZ, Itziar. Una mirada crítica al "humanitarismo" desde los estudios pospositivistas. Revista CIDOB d'Afers Internacionals, n. 117, p. 173-196, 2017.

SALAMON, Lester. The Rise of the Nonprofit Sector. Foreign Affairs, v. 73, n. 4, p. 109-122, 1994.

SALAMON, Lester; HEMS, Leslie C.; CHINNOCK, Kathryn. The Nonprofit Sector: For What and for Whom? Working Papers of the Johns Hopkins Comparative Nonprofit Sector Project, 37. Baltimore: The Johns Hopkins Center for Civil Society Studies, 2000.

SCHINDEL, Estela. Migrantes y refugiados en las fronteras de Europa. Cualificación por el sufrimiento, nuda vida y agencias paradójicas. Revista de Estudios Sociales, n. 59, p. 17-29, 2017.

SØRENSEN, Ninna Nyberg; GAMMELTOFT-HANSEN, Thomas. La comercialización de la migración internacional y los múltiples actores en la industria migratoria. E-dhc, n. 2, p. 6-23, 2013.

TICKTIN, Miriam. Los problemas de las fronteras humanitarias. Disparidades. Revista de Antropología, v. 70, n. 2, 2015. 
TREVIÑO, Javier. ¿De qué hablamos cuando hablamos de la "securitización" de la migración internacional en México?: una crítica. Foro int., v. 56, n. 2, México, p. 253-291, abr./jun 2016.

VARELA, Amarela. La "securitización" de la gubernamentalidad migratoria mediante la "externalización" de las fronteras estadounidenses a Mesoamérica. Con-temporánea, n. 5, p. 1-17, diciembre 2015.

WATSON, Scott. The 'human' as referent object?: Humanitarianism as securitization. Security Dialogue, v. 1, n. 42, p. 3-20, 2011. 